\title{
Microbial Etiology of Community Acquired Meningoencephalitis In Adults: A Retrospective Review
}

\author{
Rohit Vashisht ${ }^{1}$, Ram Gopalakrishnan ${ }^{1}$, Senthur Nambi ${ }^{1}$, Dorairajan Sureshkumar ${ }^{1}$, Nandini Sethuraman ${ }^{2}$, \\ Yamunadevi Ramanathan ${ }^{1}$, Ramasubramanian Venkatasubramanian ${ }^{1}$ \\ ${ }^{1}$ Department of Infectious disease, Apollo Hospitals, Chennai, India \\ ${ }^{2}$ Department of Microbiology, Apollo Hospitals, Chennai, India
}

\begin{abstract}
Objectives: Meningoencephalitis (ME) is a well-recognized clinical entity with diverse infectious etiologies. Only a few studies in the Indian literature are available on this issue, especially in the adult population. The aim is to study the microbial etiology of ME presenting to a tertiary care center.

Methods: A total of 97 cases labeled as ME were initially found on review of hospital records in a retrospective review over 5 years. Of these, only 62 were microbiologically confirmed and these were selected for this study.

Results: Bacteria were the cause in $21(34 \%)$ cases. Pneumococcus was the predominant bacterial etiology, found in 17 cases (detected by PCR in 15 and by culture in 3 cases). Viruses were seen in $13(21 \%)$ cases; Herpes simplex virus (HSV) was seen in 4 cases and enterovirus and varicella in 2 cases each (all were detected by PCR). We also had Arboviruses: Chikungunya (3 cases) and Dengue (2 cases) (serological diagnosis). TB was found in 15 (24\%) cases (diagnosed by positive CSF Xpert Mtb in 11 cases and by AFB culture in 7 cases). Cryptococcus was seen in another $13(21 \%)$ cases (detected by positive CSF CRAG in all cases and culture positivity in 7 cases).
\end{abstract}

Conclusion: Pneumococcus and HSV are the commonest agents causing acute ME syndrome. Mycobacterium tuberculosis and Cryptococcus accounted for all cases of chronic ME. Multiplex PCR (in acute ME) and Xpert Mtb and cryptococcal antigen (in chronic ME) play an important role in the diagnosis of culture-negative ME. J Microbiol Infect Dis 2021; 11(1):15-20.

Keywords: Meningoencephalitis, etiology, CSF, Xpert Mtb, culture

\section{INTRODUCTION}

Meningitis is characterized by the inflammation of the meninges and is identified by an abnormal number of white blood cells in cerebrospinal fluid. Encephalitis is an inflammatory process involving the brain parenchyma associated with clinical or laboratory evidence of neurological dysfunction [1]. There is often an overlap of these two syndromes in clinical practice.

Patients usually present with fever, headache, neck stiffness, and altered mental status [2]. The presentation can be acute, subacute, or chronic. The common etiological agents of acute meningoencephalitis (ME) are viruses [Japanese encephalitis virus (JEV), enteroviruses, herpes simplex] and bacteria (Streptococcus pneumoniae, Neisseria meningitidis, Haemophilus influenza). In contrast, subacute or chronic ME is usually caused by Mycobacterium tuberculosis and fungi e.g. Cryptococcus neoformans. Generally, one-third to two-thirds of encephalitis cases remain of unknown etiology despite extensive diagnostic workup [3, 4].

There are only a few studies in India looking at the specific etiological agents causing ME presenting to a tertiary health center. Hence, this study was conducted to determine the etiology and presentation of community-acquired infectious causes of ME.

\section{METHODS}

This was a retrospective descriptive study of data obtained over five years from Jan 2015 to Dec 2019. The medical records of all patients with a diagnosis of meningitis/encephalitis/ME (acute/subacute/chronic) were collected and analyzed. A total of 97 cases labeled as 
meningitis and ME were initially found on review of hospital records. The inclusion criteria for the study comprised of an age $\geq 15$ years with a diagnosis of $\mathrm{ME}$ which was based on clinical presentation, supportive CSF analysis with or without neuro-radiological findings, and a confirmed microbiological etiology. Hence out of 97 cases, 62 microbiologically confirmed cases of ME were selected for this study. A simplified algorithmic approach for guidance was devised for acute and chronic ME as shown in Figure1 and Figure 2, respectively.

Case records were reviewed to determine the demographic and clinical profile of patients along with CSF data and neuroimaging. The exclusion criteria included age below 15 years, suspected or clinically diagnosed ME without microbiologic confirmation, non-infectious encephalopathies e.g., septic/metabolic, autoimmune encephalitis, cerebral malaria, and other non-infectious mimics of altered sensorium like stroke, cortical venous thrombosis, vasculitis, and CNS space-occupying lesions.

Microbiological techniques used were microscopy and conventional bacterial, fungal and mycobacterial (Mycobacterial growth indicator tube (MGIT)) cultures. Molecular tests employed based on PCR were Xpert Mtb (for TB meningitis) (GeneXpert, Cepheid) and ME panel (for bacteria and viruses) (Biofire Film Array, bioMerieux; introduced in 2016 at our center). The CSF biomarker used was cryptococcal antigen (CRAG) (CrAg LFA- IMMY) for cryptococcal meningitis. Scrub typhus, dengue, and Chikungunya IgM were used for serologic diagnosis. (Scrub typhus IgM by ELISA by InBios kit, Dengue IgM by ELISA by Panbio kit, Chikungunya IgM by ICT with OnSite kit).

The approval of the Hospital Ethics Committee was taken prior to the start of the study.

\section{RESULTS}

Acute ME was diagnosed in $34(55 \%)$ cases and chronic ME was found in $28(45 \%)$ cases. The average age of patients was 46 years. Fever was the most common clinical finding overall, found in $25(86 \%)$ of acute cases and in 23 $(82 \%)$ of chronic ME cases. Headache was more pronounced in chronic ME cases. An altered level of consciousness on the other hand was predominantly seen in acute ME cases. Seizures were only seen in $3(10 \%)$ cases of acute ME. Neck stiffness was seen in 18 (62\%) and $16(57 \%)$ cases of acute and chronic ME respectively, on presentation to the hospital. The classic triad (defined by fever, headache, and neck rigidity) could be seen in $10(35 \%)$ cases of acute and 11 (39\%) cases of chronic ME. Out of a total of 62 patients, 12 patients were immunocompromised (8 had HIV infection, 1 was renal transplant recipient, 1 was on long-term steroids, 1 had idiopathic low CD4 count and 1 had oral cavity cancer). Diabetes was the most common co-morbid condition found in overall 17 (27\%) cases. Table 1 summarizes the above findings.

CSF abnormalities were found in nearly all cases (Table 2). Two cases of cryptococcal meningitis in PLHIV hosts had acellular CSF. One case of dengue encephalitis had a nearnormal CSF picture (white cell count $=05$, proteins $=45$, glucose $=116$ ), though she had peripheral seroconversion of $\operatorname{lgM}$ from negative to positive (Her NS-1 Ag was positive and she had systemic features of dengue). One another case of varicella encephalitis had a normal CSF picture (cell count $=05$ cells $/ \mathrm{cu} \mathrm{mm}$, proteins= $33 \mathrm{mg} / \mathrm{dl}$, glucose $=75 \mathrm{mg} / \mathrm{dl}$ ).

Table 1: Demographic and Clinical ${ }^{*}$ features

\begin{tabular}{lll}
\hline Parameter & $\begin{array}{l}\text { Acute ME } \\
(\%)\end{array}$ & $\begin{array}{l}\text { Chronic ME } \\
(\%)\end{array}$ \\
\hline $\begin{array}{l}\text { Gender (M) } \\
\text { Monthly distribution of }\end{array}$ & $20(59 \%)$ & $18(64 \%)$ \\
$\begin{array}{l}\text { acute cases } \\
\text { Maximum } \\
\text { Minimum }\end{array}$ & $\begin{array}{l}\text { October } \\
\text { June }\end{array}$ & \\
Fever & $25(86 \%)$ & $23(82 \%)$ \\
Headache & $15(52 \%)$ & $25(89 \%)$ \\
Altered sensorium & $26(89 \%)$ & $10(36 \%)$ \\
Seizures & $3(10 \%)$ & $0(0 \%)$ \\
$\begin{array}{l}\text { Neck stiffness } \\
\text { Triad of fever/ } \\
\text { headache/neck } \\
\text { rigidity }\end{array}$ & $18(62 \%)$ & $16(57 \%)$ \\
\hline${ }^{*}$ Clinical features of 5 patients were not available in records
\end{tabular}

Neuroimaging abnormalities in the form of leptomeningeal enhancement were found in 26 $(42 \%)$ cases. 
Bacteria (other than TB) were the cause of $\mathrm{ME}$ in 21 (34\%) cases, viral causes were seen in 13 (21\%) cases. TB was found in 15 (24\%) and Cryptococcus was seen in another 13(21\%) patients. Pneumococcus was the most common bacterial etiology, found in 17 (81\%) out of the 21 cases. Listeria and $H$. influenzae were seen in two $(9.5 \%)$ cases, and one $(4.7 \%)$ case, respectively.

Pneumococcus was detected by Filmarray ME panel in fifteen cases and by culture in three cases. Out of the 3 culture-positive cases, one had a positive ME panel also and in the other 2 cases, the ME panel was not done. Listeria grew in CSF culture in both cases and $H$. influenzae was detected by the ME panel. Scrub typhus meningitis was also found in one case $(4.7 \%)$ by a positive scrub lgM serology in a typical clinical setting; however, he did not have an eschar on examination.

TB meningitis was diagnosed by positive CSF Xpert Mtb in 11 cases (two were rifampicinresistant) and by AFB culture in 7 cases and by both in 3 cases (out of total 15 cases). All 13 cases of cryptococcal meningitis were positive for CSF CRAG and 7 of these cases grew cryptococcus on fungal cultures.

Of the total 13 cases of viral ME, herpes simplex virus was seen in $4(31 \%)$ cases (2 each for HSV-1 and HSV-2), followed by 2 (15.3\%) cases of enterovirus and varicella each. All of these were detected by CSF PCR. Among the arboviruses, Chikungunya (detected by CSF and serum $\lg \mathrm{M})$ constituted $3(23 \%)$ cases and dengue (detected by peripheral seroconversion) was seen in $2(15.3 \%)$ cases. Table 3 summarizes the specific bacterial and viral etiologies.

The treatment outcomes in our study based on the etiology have been depicted in Table 4.

Descriptive statistics were used to analyze discrete variables. Statistical analysis was done using Microsoft Excel. The data is represented in terms of frequency and percentages.

\section{DISCUSSION}

Meningitis and encephalitis may co-exist (as meningoencephalitis) or sometimes share similar clinical features (headache and fever) and etiologies [5]. It is important to understand the epidemiological factors and causative agents of different ME syndromes in adults, either acute or subacute/chronic, so as to initiate appropriate empiric management, especially as most Indian studies focus on the pediatric population [6]. The etiology of this overlapping syndrome is so diverse that it needs to be addressed in the current era of emerging infectious disease threats and increased diagnostic capabilities.

Pneumococcus is the main cause of bacterial meningitis in children and adults worldwide. Jayaraman et al [7] found pneumococcus to be the etiology in $82.9 \%$ of bacterial meningitis; in our study too, pneumococcus was the commonest etiological agent. In our study, PCR enabled the diagnosis of many gram stain and culture-negative cases; we suggest routinely doing PCR (or setting aside a sample for subsequent testing) in all cases, especially those with prior antibiotic exposure which may make CSF cultures sterile. Meningococcus is usually the second most common cause mainly in adolescents and young adults, though we could not find any case in our study. Listeria is an important consideration in pregnant women, the elderly, and immunocompromised persons. The incidence of $H$. influenzae meningitis has decreased now owing to increased vaccination coverage.

Viral meningitis is usually caused by enteroviruses or herpes simplex virus type 2 (HSV-2) [8], whereas a specific etiology of encephalitis had been documented in only $30.4 \%$ of cases [9]. In one of the largest Indian studies including both adults and children by Tiwari et al [10], virus identification (serologically or by molecular testing) was possible in $22.7 \%$ of acute encephalitis syndrome (AES). The most common virus identified in AES suspects in their study was HSV (8.4\%), followed by Epstein Barr virus (EBV) (5.6\%), VZV (3.1\%), Cytomegalovirus (CMV) (2.2\%), Enterovirus (EV) (1.0\%), Mumps virus (MPV) (0.9\%), Dengue virus (DV) (0.9\%). In our study, we observed that herpes virus, enterovirus, and varicella were the commonest etiologies. Among the tropical arboviruses, we had three cases of chikungunya and two cases of dengue presenting as meningoencephalitis. We had no patients with JEV in our study, possibly because of our location in a low JE prevalence urban area. We however did not do testing for JEV and 
West Nile virus with CSF and serum $\operatorname{lgM}$ as these tests were not available at our center.

Though scrub typhus is an emerging cause of encephalitis [11] and commonly presents with systemic symptoms at our center, we could find only a single patient with ME syndrome and positive serum scrub typhus IgM. Subacute or chronic meningitis is a separate entity with a different etiological spectrum. In our study, TB meningitis comprised about $25 \%$ of all cases. It is a major cause of lymphocytic predominant, low sugar, and high protein ME in an endemic setting. It is the most severe form of extrapulmonary tuberculosis and has high mortality and morbidity if there is a delay in diagnosis and treatment.
It is sometimes difficult to make a diagnosis with certainty, especially during the early phase. Acid-fast smears on CSF usually have poor sensitivity. Mycobacterial cultures especially on solid media like Lowenstein- Jensen takes about 8 weeks, though this problem has been significantly decreased by the introduction of liquid media like MGIT which can shorten the time needed to detect growth by 1 to 3 weeks. The sensitivity of CSF Xpert Mtb in the diagnosis of TB meningitis is reported as $55-60 \%$ in the literature [12] and is critically dependent on sending adequate volumes, at least $6 \mathrm{ml}$. In our study, Xpert Mtb contribution was found to be very useful, supporting its increasing role in faster and reliable diagnosis.

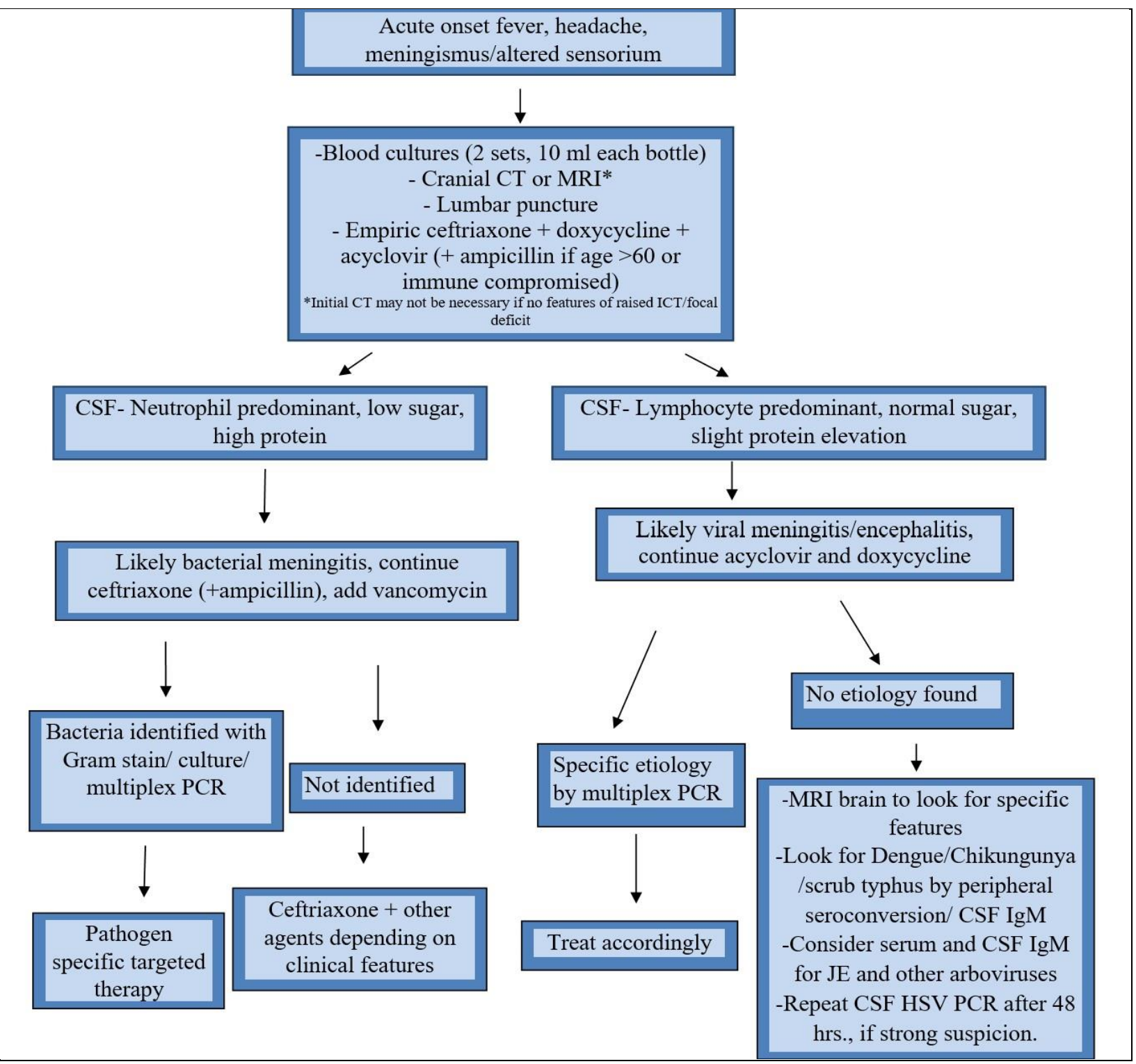

Figure 1. An approach to etiologic diagnosis of acute community acquired ME. 
Table 2. Mean values of CSF abnormalities* in each type of ME

\begin{tabular}{lccc}
\hline Etiology & Cell count (mean) & Glucose (mean) & Proteins (mean) \\
\hline Bacterial & 5481 & 45 & 396 \\
Viral & 68 & 83 & 111 \\
TB & 289 & 29 & 334 \\
Fungal & 144 & 29 & 160 \\
\hline
\end{tabular}

${ }^{*}$ CSF cell count and biochemistry of 3 patients was not available in records

Table 3. Specific bacterial and viral etiologies of acute ME cases

\begin{tabular}{lccccc}
\hline $\begin{array}{l}\text { Bacterial, } \\
(\%)\end{array}$ & $\mathrm{n}$ & $\begin{array}{c}\text { Pneumococcus, 17 } \\
(81)\end{array}$ & Listeria, 2 (9.5) & $\begin{array}{c}\text { H. influenza, } 1 \\
(4.7)\end{array}$ & $\begin{array}{c}\text { Scrub typhus, } 1 \\
(4.7)\end{array}$ \\
Viral, n (\%) & Herpes, 4 (31) & $\begin{array}{c}\text { Enterovirus, } 2 \\
(15.3)\end{array}$ & $\begin{array}{c}\text { Varicella, } 2 \\
(15.3)\end{array}$ & $\begin{array}{c}\text { Chikungunya, 3 } \\
(23)\end{array}$ & $\begin{array}{c}\text { Dengue, 2 } \\
(15.3)\end{array}$ \\
\hline
\end{tabular}

Table 4. Outcomes of various ME cases.

\begin{tabular}{lcccc}
\hline Outcome (number of cases) & Improved & Complication/sequele* & Death $^{\#}$ & Unknown $^{\$}$ \\
\hline Bacterial (21) & 14 & 4 & 1 & 2 \\
Viral (13) & 7 & 1 & 2 & 3 \\
TB (15) & 14 & 1 & 0 & 0 \\
Fungal (13) & 11 & 2 & 0 & 0
\end{tabular}

${ }^{*}$ Among the bacterial cases of ME (all pneumococcal), one developed VAP, one developed ICH, one developed SNHL, and one developed mild monoparesis. Among the viral cases, one case of varicella ME developed features of AIDP during a recovery phase. Among the TB meningitis cases, one developed ethambutol-induced optic neuritis on follow-up. Among the cases of cryptococcal $\mathrm{ME}$, one developed raised intracranial tension, and one developed hydrocephalus and later hearing loss. [VAP- ventilator associated pneumonia, ICH- intracranial hemorrhage, SNHL- sensorineural hearing loss, AIDP- acute inflammatory demyelinating polyneuropathy]

\# 1 death occurred in Pneumococal and 2 in chikungunya encephalitis (all sepsis were geriatric patients)

$\$$ These cases could not be followed up as required data was not available.

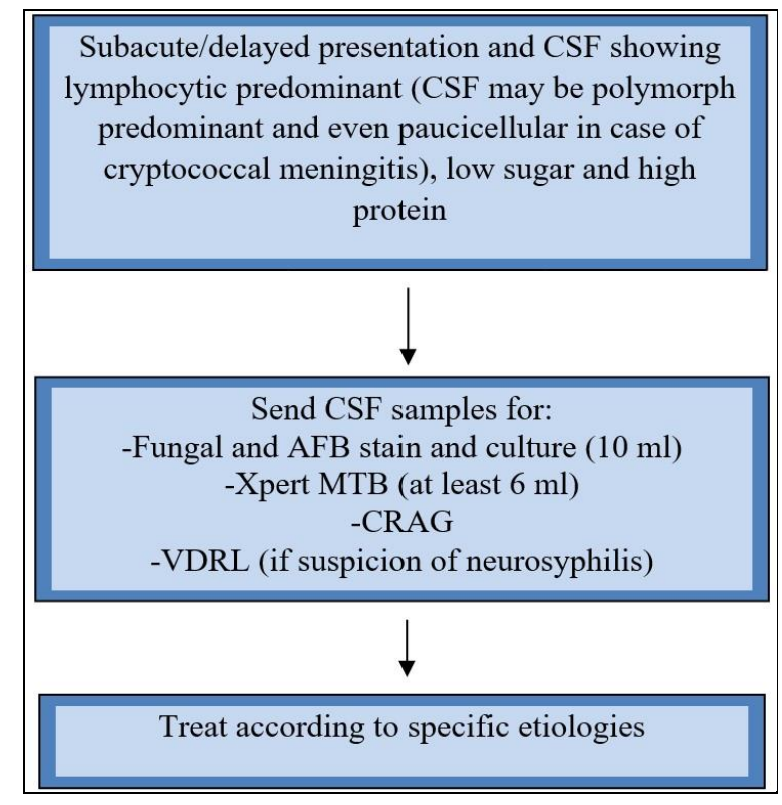

Fig 2. An algorithm for etiologic diagnosis of chronic community acquired ME.

A significant proportion of chronic ME was caused by cryptococcus ( $21 \%$ of total cases). Unlike C. gatti, C. neoformans is a rare cause of ME in immunocompetent individuals [13]. In our study also, three out of 13 cases caused by Cryptococcus neoformans were found in immunocompetent individuals, with the remaining in HIV-positive patients. Another interesting finding is acellular CSF which was found in two of our cryptococcal cases, also well known in the literature [14].

Cryptococcal antigen (CRAG) can be detected using latex agglutination, which historically has a sensitivity and specificity of $>99 \%$ in blood and CSF $[15,16]$. In our study, CSF CRAG was found in all the cases, with cultures positive 
proven in seven of 13 cases. We recommend that CRAG should be routinely sent for all subacute or chronic ME.

The main strength of our study was that we studied only etiologically confirmed cases and excluded all possible or probable ME based on imaging or CSF formula.

The main limitations were the retrospective nature and small sample size of the study which was due to exclusion of all ME cases without microbiological confirmation of the etiology.

\section{Conclusion}

Pneumococcus is the commonest bacterial pathogen in acute ME and multiplex PCR is valuable in diagnosis, especially in culturenegative cases or patients on prior antibiotics. Herpes simplex was the commonest viral etiology. ME panel is emerging as an important tool to discern the etiology of acute ME, and we recommend that it be sent (or a sample saved for later testing) for all patients with acute ME.

\section{ACKNOWLEDGMENTS}

Declaration of Conflicting Interests: The authors declare that they have no conflict of interest.

\section{Funding: Not applicable \\ REFERENCES}

1. Beckham JD, Tyler KL. Encephalitis. In: Bennett JE, Dolin R, Blaser MJ, eds. Mandell, Douglas and Bennett's: Principles and Practice of Infectious Diseases, 9th Edition. Philadelphia: Elsevier, 2019: 1226-1247.

2. Mehrdadi S. Acute Bacterial Meningitis: Diagnosis, Treatment and Prevention. J Arch Mil Med 2019; 6(4): e84749. doi: 10.5812/jamm.84749.

3. Glaser CA, Gilliam S, Schnurr D, et al. In search of encephalitis etiologies: diagnostic challenges in the California Encephalitis Project, 1998-2000. Clin Infect Dis 2003; 36:731-742.

4. Glaser CA. Honarmand S, Anderson LJ, et al. Beyond viruses: Clinical profiles and etiologies associated with encephalitis. Clin Infect Dis 2006; 43:1565-1577.

5. Beaman MH. Community-acquired acute meningitis and encephalitis: a narrative review. Med J Aust 2018; 209(10):449-454.

6. Yerramilli A, Mangapati P, Prabhakar S, Sirimulla $\mathrm{H}$, Vanam S, Voora Y. A study on the clinical outcomes and management of meningitis at a tertiary care centre. Neurol India 2017; 65: 1006-12.
7. Jayaraman $\mathrm{Y}$, Veeraraghavan B, Chethrapilli Purushothaman GK, et al. Burden of bacterial meningitis in India: Preliminary data from a hospitalbased sentinel surveillance network. PLOS ONE 2018; 13(5): e0197198.

8. McGill F, Griffiths MJ, Solomon T. Viral meningitis: current issues in diagnosis and treatment. Curr Opin Infect Dis 2017; 30: 248-256.

9. Huppatz C, Durrheim DN, Levi C, et al. Etiology of encephalitis in Australia, 1990-2007. Emerg Infect Dis 2009; 15(9): 1359-1365.

10. Tiwari JK, Malhotra B, Chauhan A, et al. Aetiological study of viruses causing acute encephalitis syndrome in North West India. Indian J Med Microbiol 2017; 35:529-534.

11. Viswanathan S, Muthu V, Iqbal N, Remalayam B, George T. Scrub Typhus Meningitis in South India - A Retrospective Study. PLoS One 2013; 8(6): e66595.

12. Nhu NT, Heemskerk D, Thu do DA, et al. Evaluation of GeneXpert MTB/RIF for Diagnosis of Tuberculous Meningitis. J Clin Microbiol 2014; 52(1):226-33

13. Mada P, Nowack B, Cady B, Joel Chandranesan. Disseminated cryptococcosis in an immunocompetent patient. BMJ Case Rep 2017. doi: 10.1136/bcr-2016218461.

14. Koshy JM, Mohan S, Deodhar D, John M, Oberoi A, Pannu A. Clinical Diversity of CNS Cryptococcosis. J Assoc Physicians India 2016; 64(10):15-19.

15. Rajasingham R, Wake RM, Beyene T, Katende A, Letang $E$, Boulware DR. Cryptococcal Meningitis Diagnostics and Screening in the Era of Point -ofCare Laboratory Testing. J. Clin. Microbiol 2019; 57(1): e01238-18.

16. Boulware DR, Rolfes MA, Rajasingham R, et al. Multisite validation of cryptococcal antigen lateral flow assay and quantification by laser thermal contrast. Emerg Infect Dis 2014; 20(1): 45 -53. 